\title{
Novel Audio Features for Music Emotion Recognition
}

\author{
Renato Panda ${ }^{\circledR}$, Ricardo Malheiro ${ }^{\circledR}$, and Rui Pedro Paiva ${ }^{\circledR}$
}

\begin{abstract}
This work advances the music emotion recognition state-of-the-art by proposing novel emotionally-relevant audio features. We reviewed the existing audio features implemented in well-known frameworks and their relationships with the eight commonly defined musical concepts. This knowledge helped uncover musical concepts lacking computational extractors, to which we propose algorithms - namely related with musical texture and expressive techniques. To evaluate our work, we created a public dataset of 900 audio clips, with subjective annotations following Russell's emotion quadrants. The existent audio features (baseline) and the proposed features (novel) were tested using 20 repetitions of 10 -fold cross-validation. Adding the proposed features improved the F1-score to 76.4 percent (by 9 percent), when compared to a similar number of baseline-only features. Moreover, analysing the features relevance and results uncovered interesting relations, namely the weight of specific features and musical concepts to each emotion quadrant, and warrant promising new directions for future research in the field of music emotion recognition, interactive media, and novel music interfaces.
\end{abstract}

Index Terms-Affective computing, audio databases, emotion recognition, feature extraction, music information retrieval

\section{INTRODUCTION}

TN recent years, Music Emotion Recognition (MER) has 1 attracted increasing attention from the Music Information Retrieval (MIR) research community. Presently, there is already a significant corpus of research works on different perspectives of MER, e.g., classification of song excerpts [1], [2], emotion variation detection [3], automatic playlist generation [4], exploitation of lyrical information [5] and bimodal approaches [6]. However, several limitations still persist, namely, the lack of a consensual and public dataset and the need to further exploit emotionally-relevant acoustic features. Particularly, we believe that features specifically suited to emotion detection are needed to narrow the socalled semantic gap [7] and their absence hinders the progress of research on MER. Moreover, existing system implementation shows that the state-of-the-art solutions are still unable to accurately solve simple problems, such as classification with few emotion classes (e.g., four to five). This is supported by both existing studies [8], [9] and the small improvements in the results attained in the 2007-2017 MIREX Audio Mood Classification (AMC) task ${ }^{1}$, an annual comparison of MER algorithms. These system implementations and

1. http://www.music-ir.org/mirex/

- R. Panda and R. P. Paiva are with the Center for Informatics and Systems of the University of Coimbra (CISUC), Coimbra 3004-531, Portugal. E-mail: \{panda, ruipedro\}@dei.uc.pt.

- R. Malheiro is with Center for Informatics and Systems of the University of Coimbra (CISUC) and Miguel Torga Higher Institute, Coimbra 3000132, Portugal.E-mail: rsmal@dei.uc.pt.

Manuscript received 10 Jan. 2018; revised 21 Mar. 2018; accepted 24 Mar. 2018. Date of publication 0 . 0000; date of current version 0 . 0000.

(Coressponding author: Renato Panda).

Recommended for acceptance by Y.-H. Yang.

For information on obtaining reprints of this article, please send e-mail to: reprints@ieee.org, and reference the Digital Object Identifier below.

Digital Object Identifier no. 10.1109/TAFFC.2018.2820691 research results show a glass ceiling in MER system perform- 37 ances [7].

Several factors contribute to this glass ceiling of MER sys- 39 tems. To begin with, our perception of emotion is inherently 40 subjective: different people may perceive different, even 41 opposite, emotions when listening to the same song. Even 42 when there is an agreement between listeners, there is often 43 ambiguity in the terms used regarding emotion description 44 and classification [10]. It is not well-understood how and why 45 some musical elements elicit specific emotional responses in 46 listeners [10].

Second, creating robust algorithms to accurately capture 48 these music-emotion relations is a complex problem, involv- 49 ing, among others, tasks such as tempo and melody estima- 50 tion, which still have much room for improvement.

Third, as opposed to other information retrieval problems, 52 there are no public, widely accepted and adequately vali- 53 dated, benchmarks to compare works. Typically, researchers 54 use private datasets (e.g., [11]) or provide only audio features 55 (e.g., [12]). Even though the MIREX AMC task has contrib- 56 uted with one dataset to alleviate this problem, several major 57 issues have been identified in the literature. Namely, the 58 defined taxonomy lacks support from music psychology and 59 some of the clusters show semantic and acoustic overlap [2]. 60

Finally, and most importantly, many of the audio fea- 61 tures applied in MER were created for other audio recogni- 62 tion applications and often lack emotional relevance. 63 Hence, our main working hypothesis is that, to further 64 advance the audio MER field, research needs to focus on 65 what we believe is its main, crucial, and current problem: to 66 capture the emotional content conveyed in music through 67 better designed audio features.

This raises the core question we aim to tackle in this 69 paper: which features are important to capture the emo- 70 tional content in a song? Our efforts to answering this 71 
TABLE 1

Musical Features Relevant to MER

\begin{tabular}{ll}
\hline Features & \multicolumn{1}{c}{ Examples } \\
\hline Timing & Tempo, tempo, variation, duration, contrast. \\
Dynamics & Overall level, crescendo/decrescendo, accents. \\
Articulation & Overall (staccato, legato), variability. \\
Timbre & Spectral richness, harmonic richness. \\
Pitch & High or low. \\
Interval & Small or large. \\
Melody & Range (small or large), direction (up or down). \\
Tonality & Chromatic-atonal, key-oriented. \\
Rhythm & Regular, irregular, smooth, firm, flowing, rough. \\
Mode & Major or minor. \\
Loudness & High or low. \\
Musical form & Complexity, repetition, disruption. \\
Vibrato & Extent, range, speed. \\
\hline
\end{tabular}

question required: i) a review of computational audio features currently implemented and available in the state-ofthe-art audio processing frameworks; ii) the implementation and validation of novel audio features (e.g., related with music performance expressive techniques or musical texture).

Additionally, to validate our work, we have constructed a dataset that we believe is better suited to the current situation and problem: it employs four emotional classes, from the Russell's emotion circumplex [13], avoiding both unvalidated and overly complex taxonomies; it is built with a semi-automatic method (AllMusic annotations, along with simpler human validation), to reduce the resources required to build a fully manual dataset.

Our classification experiments showed an improvement of 9 percent in F1-Score when using the top 100 baseline and novel features, while compared to the top 100 baseline features only. Moreover, even when the top 800 baseline features is employed, the result is 4.7 percent below the one obtained with the top 100 baseline and novel features set.

This paper is organized as follows. Section 2 reviews the related work. Section 3 presents a review of the musical concepts and related state-of-the-art audio features, as well as the employed methods, from dataset acquisition to the novel audio features and the classification strategies. In Section 4, experimental results are discussed. Finally, conclusions and possible directions for future work are included in Section 5.

\section{Related Work}

Musical Psychology researchers have been actively studying the relations between music and emotions for decades. In this process, different emotion paradigms (e.g., categorical or dimensional) and related taxonomies (e.g., Hevner, Russell) have been developed [13], [14] and exploited in different computational MER systems, e.g., [1], [2], [3], [4], [5], [6], [10], [11], [15], [16], [17], [18], [19], along with specific MER datasets, e.g., [10], [16], [19].

Emotion in music can be studied as: i) perceived, as in the emotion an individual identifies when listening; ii) felt, regarding the emotional response a user feels when listening, which can be different from the perceived one; iii) or transmitted, representing the emotion that the performer or composer aimed to convey. As mentioned, we focus this 114 work on perceived emotion.

Regarding the relations between emotions and specific 116 musical attributes, several studies uncovered interesting 117 associations. As an example: major modes are frequently 118 related to emotional states such as happiness or solemnity, 119 whereas minor modes are often associated with sadness or 120 anger [20]; simple, consonant, harmonies are usually happy, 121 pleasant or relaxed. On the contrary, complex, dissonant, 122 harmonies relate to emotions such as excitement, tension or 123 sadness, as they create instability in a musical motion [21]. 124 Moreover, researchers identified many musical features 125 related to emotion, namely: timing, dynamics, articulation, 126 timbre, pitch, interval, melody, harmony, tonality, rhythm, 127 mode, loudness, vibrato, or musical form [11], [21], [22], 128 [23]. A summary of musical characteristics relevant to emo- 129 tion is presented in Table 1.

Despite the identification of these relations, many of 131 them are not fully understood, still requiring further musi- 132 cological and psychological studies, while others are diffi- 133 cult to extract from audio signals. Nevertheless, several 134 computational audio features have been proposed over the 135 years. While the number of existent audio features is high, 136 many were developed to solve other problems (e.g., Mel- 137 frequency cepstral coefficients (MFCCs) for speech recogni- 138 tion) and may not be directly relevant to MER. 139

Nowadays, most proposed audio features are imple- 140 mented and available in audio frameworks. In Table 2, we 141 summarize several of the current state-of-the-art (hereafter 142 termed standard) audio features, available in widely 143 adopted frameworks, namely, the MIR Toolbox [24], Mar- 144 syas [25] and PsySound3 [26].

Musical attributes are usually organized into four to 146 eight different categories (depending on the author, e.g., 147 [27], [28]), each representing a core concept. Here, we follow 148 an eight categories organization, employing rhythm, 149 dynamics, expressive techniques, melody, harmony, tone 150 colour (related to timbre), musical texture and musical 151 form. Through this organization, we are able to better 152 understand: i) where features related to emotion belong; ii) 153 and which categories may lack computational models to 154 extract musical features relevant to emotion. 155

One of the conclusions obtained is that the majority of avail- 156 able features are related with tone colour (63.7 percent). Also, 157 many of these features are abstract and very low-level, captur- 158 ing statistics about the waveform signal or the spectrum. 159 These are not directly related with the higher-level musical 160 concepts described earlier. As an example, MFCCs belong to 161 tone colour but do not give explicit information about the 162 source or material of the sound. Nonetheless, they can implic- 163 itly help to distinguish these. This is an example of the men- 164 tioned semantic gap, where high level concepts are not being 165 captured explicitly with the existent low level features. 166

This agrees with the conclusions presented in [8], [9], 167 where, among other things, the influence of the existent 168 audio features to MER was assessed. Results of previous 169 experiments showed that "the used spectral features out- 170 performed those based on rhythm, dynamics, and, to a 171 lesser extent, harmony" [9]. This supports the idea that 172 more adequate audio features related to some musical con- 173 cepts are lacking. In addition, the number of implemented 174 
TABLE 2

\section{Summary of Standard Audio Features}

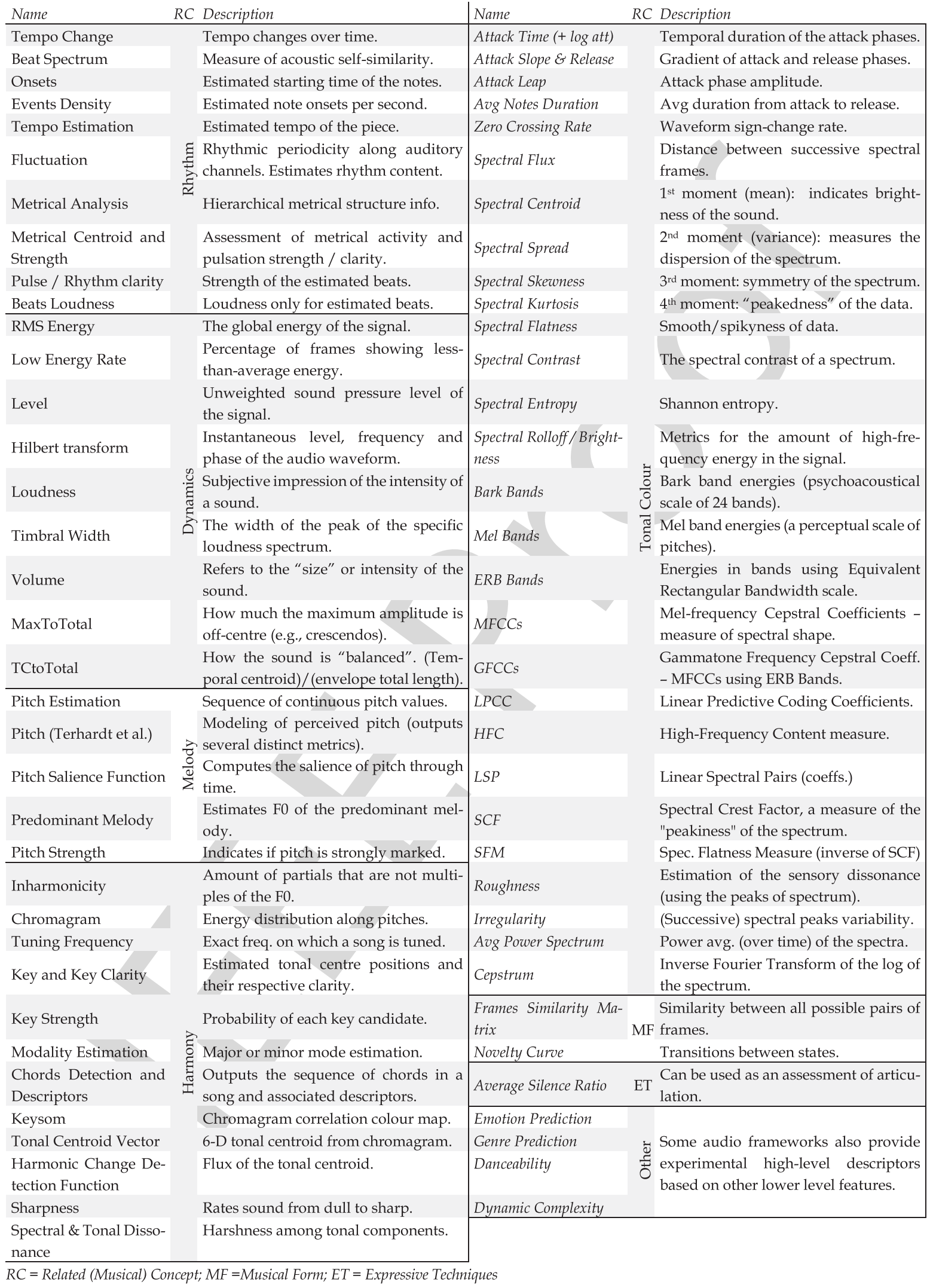

audio features is highly unproportional, with nearly 60 percent in the cited article belonging to timbre (spectral) [9].

In fact, very few features are mainly related with expressive techniques, musical texture (which has none) or musical form. Thus, there is a need for audio features esti- 179 mating higher-level concepts, e.g., expressive techniques 180 and ornamentations like vibratos, tremolos or staccatos 181 (articulation), texture information such as the number of 182 


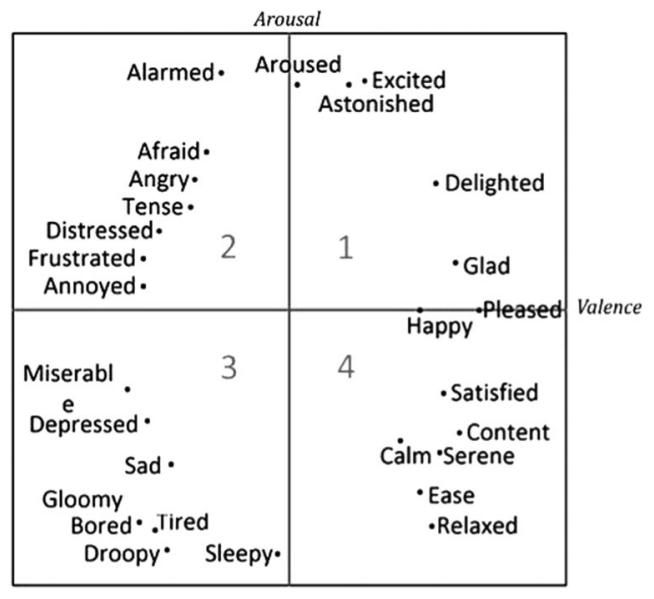

Fig. 1. Russell's circumplex model of emotion (adapted from [9]). musical lines or repetition and complexity in musical form. Concepts such as rhythm, melody, dynamics and harmony already have some related audio features available. The main question is: are they enough to the problem? In the next sections we address these questions by proposing novel high-level audio features and running classification experiments with both existent and novel features.

To conclude, the majority of current computational MER works (e.g., [3], [10], [16]) share common limitations such as low to average results, especially regarding valence, due to the aforesaid lack of relevant features; lack of uniformity in the selected taxonomies and datasets, which makes it impossible to compare different approaches; and the usage of private datasets, unavailable to other researchers for benchmarking. Additional publicly available datasets exist, most suffering from the same previously described problems, such as: i) Million Song Dataset, which covers a high number of songs but providing only features, metadata and uncontrolled annotations (e.g., based on social media information such as Last. FM) [12]; ii) MoodSwings, which has a limited number of samples [29]; iii) Emotify, which is focused on induced rather than perceived emotions [30]; iv) MIREX, which employs unsupported taxonomies and contains overlaps between clusters [31]; v) DEAM, which is sizeable but shows low agreement between annotators, as well as issues such as noisy clips (e.g., claps, speak, silences) or clear variations in emotion in supposedly static excerpts [32]; vi) or existent datasets, which still require manual verification of the gathered annotations or clips quality, such as [6].

\section{Methods}

In this section we introduce the proposed novel audio features and describe the emotion classification experiments carried out. To assess this, and given the mentioned limitations of available datasets, we started by building a newer dataset that suits our purposes.

\subsection{Dataset Acquisition}

The currently available datasets have several issues, as discussed in Section 2. To avoid these pitfalls, the following objectives were pursued to build ours:

1) Use a simple taxonomy, supported by psychological studies. In fact, current MER research is still unable to properly solve simpler problems with high accu- 224 racy. Thus, in our opinion, there are few advantages 225 to currently tackle problems with higher granularity, 226 where a high number of emotion categories or con- 227 tinuous values are used;

2) Perform semi-automatic construction, reducing the 229 resources needed to build a sizeable dataset; $\quad 230$

3) Obtain a medium-high size dataset, containing hun- 231 dreds of songs;

4) Create a public dataset prepared to further research 233 works, thus providing emotion quadrants as well as 234 genre, artists or emotion tags for multi-label 235 classification; 236

Regarding emotion taxonomies, several distinct models 237 have been proposed over the years, divided into two major 238 groups: categorical and dimensional. It is often argued that 239 dimensional paradigms lead to lower ambiguity, since 240 instead of having a discrete set of emotion adjectives, emo- 241 tions are regarded as a continuum [10]. A widely accepted 242 dimensional model in MER is James Russell's [13] circum- 243 plex model. There, Russell affirms that each emotional state 244 sprouts from two independent neurophysiologic systems. 245 The two proposed dimensions are valence (pleasant- 246 unpleasant) and activity or arousal (aroused-not aroused), 247 or AV. The resulting two-dimensional plane forms four dif- 248 ferent quadrants: 1- exuberance, 2- anxiety, 3- depression 249 and 4- contentment (Fig. 1). Here, we follow this taxonomy. 250

The AllMusic API ${ }^{2}$ served as the source of musical infor- 251 mation, providing metadata such as artist, title, genre and 252 emotion information, as well as 30-second audio clips for 253 most songs. The steps for the construction of the dataset are 254 described in the following paragraphs. 255

Step 1: AllMusic API querying. First, we queried the API for 257 the top songs for each of the 289 distinct emotion 258 tags in it. This resulted in 370611 song entries, of 259 which 89 percent had an associated audio sample 260 and 98 percent had genre tags, with 28646 distinct 261 artist tags present. These 289 emotion tags used by 262 AllMusic are not part of any known supported tax- 263 onomy, still are said to be "created and assigned to 264 music works by professional editors" [33]. 265

Step 2: Mapping of AllMusic tags into quadrants. Next, we use 267 the Warriner's adjectives list [34] to map the 289 All- 268 Music tags into Russell's AV quadrants. Warriner's 269 list contains 13915 English words with affective rat- 270 ings in terms of arousal, valence and dominance 271 (AVD). It is an improvement over previous studies 272 (e.g., ANEW adjectives list [35]), with a better docu- 273 mented annotation process and a more comprehen- 274 sive list of words. Intersecting Warriner and 275 AllMusic tags results in 200 common words, where a 276 higher number have positive valence (Q1: 49, Q2: 35, 277 Q3: 33, Q4: 75).

Step 3: Processing and filtering. Then, the set of related meta- 280 data, audio clips and emotion tags with AVD values 281 was processed and filtered. As abovementioned, in 282

2. http://developer.rovicorp.com/docs 
our dataset each song is annotated according to one of Russell's quadrants. Hence, the first iteration consisted in removing song entries where a dominant quadrant was not present. We defined a quadrant to be dominant when at least 50 percent of the emotion tags of the song belong to it. This reduced the set to 120733 song entries. Further cleaning was performed by removing duplicated song entries using approximate string matching. A second iteration removed any song entry without genre information and having less than 3 emotion tags associated to meet the predefined objectives, reducing the set to 39983 entries. Then, a third iteration was used to deal with the unbalanced nature of the original data in terms of emotion tags and genres. Finally, the dataset was sub-sampled, resulting in a candidate set containing 2200 song clips, balanced in terms of quadrants and genres in each quadrant, which was then manually validated, as described in the next section.

\subsection{Validation of Emotion Annotations}

Not many details are known regarding the AllMusic emotion tagging process, apart from supposedly being made by experts [33]. It is unclear whether they are annotating songs using only audio, lyrics or a combination of both. In addition, it is unknown how the 30-second clips that represent each song are selected by AllMusic. In our analysis, we observed several noisy clips (e.g., containing applauses, only speech, long silences, inadequate song segments such as the introduction).

Hence, a manual blind inspection of the candidate set was conducted. Subjects were given sets of randomly distributed clips and asked to annotate them accordingly in terms of Russell's quadrants. Beyond selecting a quadrant, the annotation framework allowed subjects to mark clips as unclear, if the emotion was unclear to the subject, or bad, if the clip contained noise (as defined above).

To construct the final dataset, song entries with clips considered bad or where subjects' and AllMusic's annotations did not match were excluded. The quadrants were also rebalanced to obtain a final set of 900 song entries, with exactly 225 for each quadrant. In our opinion, the dataset dimension is an acceptable compromise between having a bigger dataset using tools such as the Amazon Mechanical Turk or automatic but uncontrolled sources as annotations, and a very small and resource intensive dataset annotated exclusively by a high number of subjects in a controlled environment.

Each song entry is tagged in terms of Russell's quadrants, arousal and valence classes (positive or negative), and multi-label emotion tags. In addition, emotion tags have an associated AV value from Warriner's list, which can be used to place songs in the AV plane, allowing the use of this dataset in regression problems (yet to be demonstrated). Moreover, the remaining metadata (e.g., title, artist, album, year, genre and theme) can also be exploited in other MIR tasks. The final dataset is publicly available in our site ${ }^{3}$.

\subsection{Standard Audio Features}

As abovementioned, frameworks such as the MIR Toolbox, Marsyas and PsySound offer a large number of

3. http://mir.dei.uc.pt/resources/MER_audio_taffc_dataset.zip computational audio features. In this work, we extract a 340 total of 1702 features from those three frameworks. This 341 high amount of features is also because several statistical 342 measures were computed for time series data. 343

Afterwards, a feature reduction stage was carried to dis- 344 card redundant features obtained by similar algorithms 345 across the selected audio frameworks. This process con- 346 sisted in the removal of features with correlation higher 347 than 0.9, where features with lower weight were discarded, 348 according to the ReliefF [36] feature selection algorithm. 349 Moreover, features with zero standard deviation were also 350 removed. As a result, the number of baseline features was 351 reduced to 898. A similar feature reduction process was car- 352 ried out with the novel features presented in the following 353 subsection.

These standard audio features serve to build baseline 355 models against which new approaches, employing the 356 novel audio features proposed in the next section, can be 357 benchmarked. The illustrated number of novel features is 358 described as follows.

\subsection{Novel Audio Features}

Many of the standard audio features are low-level, extracted 361 directly from the audio waveform or the spectrum. How- 362 ever, we naturally rely on clues like melodic lines, notes, 363 intervals and scores to assess higher-level musical concepts 364 such as harmony, melody, articulation or texture. The 365 explicit determination of musical notes, frequency and 366 intensity contours are important mechanisms to capture 367 such information and, therefore, we describe this prelimi- 368 nary step before presenting actual features, as follows. 369

\subsubsection{From the Audio Signal to MIDI Notes}

Going from audio waveform to music score is still an 371 unsolved problem, and automatic music transcription algo- 372 rithms are still imperfect [37]. Still, we believe that estimat- 373 ing things such as predominant melody lines, even if 374 imperfect, give us relevant information that is currently 375 unused in MER.

To this end, we built on previous works by Salomon et al. 377 [38] and Dressler [39] to estimate predominant fundamental 378 frequencies (f0) and saliences. Typically, the process starts 379 by identifying which frequencies are present in the signal at 380 each point in time (sinusoid extraction). Here, $46.44 \mathrm{msec} 381$ (1024 samples) frames with $5.8 \mathrm{msec}$ (128 samples) hopsize 382 (hereafter denoted hop) were selected. 383

Next, harmonic summation is used to estimate the 384 pitches in these instants and how salient they are (obtaining 385 a pitch salience function). Given this, the series of consecu- 386 tive pitches which are continuous in frequency are used to 387 form pitch contours. These represent notes or phrases. 388 Finally, a set of computations is used to select the f0s that 389 are part of the predominant melody [38]. The resulting pitch 390 trajectories are then segmented into individual MIDI notes 391 following the work by Paiva et al. [40]. 392

Each of the $N$ obtained notes, hereafter denoted as note $e_{i}, 393$ is characterized by: the respective sequence of f0s (a total of 394 $L_{i}$ frames), $f 0_{j, i}, j=1,2, \ldots L_{i}$; the corresponding MIDI 395 note numbers (for each $\mathrm{f} 0$ ), $m i d i_{j, i} ;$ the overall MIDI note 396 value (for the entire note), $M I D I_{i}$; the sequence of pitch sali- 397 ences, $s a l_{j, i}$; the note duration, $n d_{i}(\mathrm{sec})$; starting time, $s t_{i} 398$ 
(sec); and ending time, $e t_{i}(\mathrm{sec})$. This information is exploited to model higher level concepts such as vibrato, glissando, articulations and others, as follows.

In addition to the predominant melody, music is composed of several melodic lines produced by distinct sources. Although less reliable, there are works approaching multiple (also known as polyphonic) F0 contours estimation from these constituent sources. We use Dressler's multi-F0 approach [39] to obtain a framewise sequence of fundamental frequencies estimates.

\subsubsection{Melodic Features}

Melody is a key concept in music, defined as the horizontal succession of pitches. This set of features consists in metrics obtained from the notes of the melodic trajectory.

MIDI Note Number (MNN) statistics. Based on the MIDI note number of each note, $M I D I_{i}$ (see Section 3.4.1), we compute 6 statistics: MIDImean, i.e., the average MIDI note number of all notes, MIDIstd (standard), MIDIskew (skewness), MIDIkurt (kurtosis), MIDImax (maximum) and MIDImin (minimum).

Note Space Length (NSL) and Chroma NSL (CNSL). We also extract the total number of unique MIDI note values, $N S L$, used in the entire clip, based on $M I D I_{i}$. In addition, a similar metric, chroma NSL, $C N S L$, is computed, this time mapping all MIDI note numbers to a single octave (result 1 to 12).

Register Distribution. This class of features indicates how the notes of the predominant melody are distributed across different pitch ranges. Each instrument and voice type has different ranges, which in many cases overlap. In our implementation, 6 classes were selected, based on the vocal categories and ranges for non-classical singers [41]. The resulting metrics are the percentage of MIDI note values in the melody, $M I D I_{i}$, that are in each of the following registers: Soprano (C4-C6), Mezzo-soprano (A3-A5), Contralto (F3-E5), Tenor (B2-A4), Baritone (G2-F4) and Bass (E2-E4). For instance, for soprano, it comes $(1)^{4}$ :

$$
R \text { Dsoprano }=\frac{\sum_{i=1}^{N}\left[72 \leq M I D I_{i} \leq 96\right]}{N} .
$$

Register Distribution per Second. In addition to the previous class of features, these are computed as the ratio of the sum of the duration of notes with a specific pitch range (e.g., soprano) to the total duration of all notes. The same 6 pitch range classes are used.

Ratios of Pitch Transitions. Music is usually composed of sequences of notes of different pitches. Each note is followed by either a higher, lower or equal pitch note. These changes are related with the concept of melody contour and movement. They are also important to understand if a melody is conjunct (smooth) or disjunct. To explore this, the extracted MIDI note values are used to build a sequence of transitions to higher, lower and equal notes.

The obtained sequence marking transitions to higher, equal or lower notes is summarized in several metrics, namely: Transitions to Higher Pitch Notes Ratio (THPNR), Transitions to Lower Pitch Notes Ratio (TLPNR) and Transitions to Equal Pitch Notes Ratio (TEPNR). There, the ratio of

4. Using the Iverson bracket notation. the number of specific transitions to the total number of 456 transitions is computed. Illustrating for THPNR, (2): 457

$$
T H P N R=\frac{\sum_{i=1}^{N-1}\left[M I D I_{i}<M I D I_{i+1}\right]}{N-1} .
$$

Note Smoothness (NS) statistics. Also related to the charac- 461 teristics of the melody contour, the note smoothness feature 462 is an indicator of how close consecutive notes are, i.e., how 463 smooth is the melody contour. To this end, the difference 464 between consecutive notes (MIDI values) is computed. The 465 usual 6 statistics are also calculated.

$$
N \text { Smean }=\frac{\sum_{i=1}^{N-1}\left|M I D I_{i+1}-M I D I_{i}\right|}{N-1} .
$$

\subsubsection{Dynamics Features}

Exploring the pitch salience of each note and how it com- 471 pares with neighbour notes in the score gives us informa- 472 tion about their individual intensity, as well as and 473 intensity variation. To capture this, notes are classified as 474 high (strong), medium and low (smooth) intensity based on 475 the mean and standard deviation of all notes, as in (4):

$$
\begin{aligned}
S A L_{i} & =\operatorname{median}_{1 \leq j \leq L_{i}}\left(s a l_{j, i}\right) \\
\mu_{s} & =\operatorname{mean}_{1 \leq i \leq N}\left(S A L_{i}\right) \\
\sigma_{s} & =\operatorname{std}_{1 \leq i \leq N}\left(S A L_{i}\right) \\
I N T_{i} & = \begin{cases}\text { low, } & S A L_{i} \leq \mu_{s}-0.5 \sigma_{s} \\
\text { medium, } & \mu_{s}-0.5 \sigma_{s}<S A L_{i}<\mu_{s}+0.5 \sigma_{s} \\
\text { high, } & S A L_{i} \geq \mu_{s}+0.5 \sigma_{s}\end{cases}
\end{aligned}
$$

(4) 478

There, $S A L_{i}$ denotes the median intensity of note $_{i}$, for all 480 its frames and $I N T_{i}$ stands for the qualitative intensity of 481 the same note. Based on the calculations in (4), the following 482 features are extracted.

Note Intensity (NI) statistics. Based on the median pitch 484 salience of each note, we compute same 6 statistics. 485

Note Intensity Distribution. This class of features indicates 486 how the notes of the predominant melody are distributed 487 across the three intensity ranges defined above. Here, we 488 define three ratios: Low Intensity Notes Ratio (LINR), 489 Medium Intensity Notes Ratio (MINR) and High Intensity 490 Notes Ratio (HINR). These features indicate the ratio of 491 number of notes with a specific intensity (e.g., low intensity 492 notes, as defined above) to the total number of notes.

493

Note Intensity Distribution per Second. Low Intensity Note 494 Duration Ratio (LINDR), Medium Intensity Notes Duration 495 Ratio (MINDR) and High Intensity Notes Duration Ratio 496 (HINDR) statistics. These features are computed as the ratio 497 of the sum of the duration of notes with a specific intensity 498 to the total duration of all notes. Furthermore, the usual 6499 statistics are calculated.

Ratios of Note Intensity Transitions. Transitions to Higher 501 Intensity Notes Ratio (THINR), Transitions to Lower Inten- 502 sity Notes Ratio (TLINR) and Transitions to Equal Intensity 503 Notes Ratio (TELNR). In addition to the previous metrics, 504 these features capture information about changes in note 505 
dynamics by measuring the intensity differences between consecutive notes (e.g., the ratio of transitions from low to high intensity notes).

Crescendo and Decrescendo (CD) statistics. Some instruments (e.g., flute) allow intensity variations in a single note. We identify notes as having crescendo or decrescendo (also known as diminuendo) based on the intensity difference between the first half and the second half of the note. A threshold of 20 percent variation between the median of the two parts was selected after experimental tests. From these, we compute the number of crescendo and decrescendo notes (per note and per sec). In addition, we compute sequences of notes with increasing or decreasing intensity, computing the number of sequences for both cases (per note and per sec) and length crescendo sequences in notes and in seconds, using the 6 previously mentioned statistics.

\subsubsection{Rhythmic Features}

Music is composed of sequences of notes changing over time, each with a specific duration. Hence, statistics on note durations are obvious metrics to compute. Moreover, to capture the dynamics of these durations and their changes, three possible categories are considered: short, medium and long notes. As before, such ranges are defined according to the mean and standard deviation of the duration of all notes, as in (5). There, $N D_{i}$ denotes the qualitative duration of note $e_{i}$.

$$
\begin{aligned}
\mu_{d} & =\operatorname{mean}_{1 \leq i \leq N}\left(n d_{i}\right) \\
\sigma_{d} & =\operatorname{std}_{1 \leq i \leq N}\left(n d_{i}\right) \\
N D_{i} & = \begin{cases}\text { short, } & n d_{i} \leq \mu_{d}-0.5 \sigma_{d} \\
\text { medium, } & \mu_{d}-0.5 \sigma_{d}<n d_{i}<\mu_{d}+0.5 \sigma_{d} . \\
\text { long, } & n d_{i} \geq \mu_{d}+0.5 \sigma_{d}\end{cases}
\end{aligned}
$$

The following features are then defined.

Note Duration (ND) statistics. Based on the duration of each note, $n d_{i}$ (see Section 3.4.1), we compute the usual 6 statistics.

Note Duration Distribution. Short Notes Ratio (SNR), Medium Length Notes Ratio (MLNR), Long Notes Ratio (LNR). These features indicate the ratio of the number of notes in each category (e.g., short duration notes) to the total number of notes.

Note Duration Distribution per Second. Short Notes Duration Ratio (SNDR), Medium Length Notes Duration Ratio (MLNDR) and Long Notes Duration Ratio (LNDR) statistics. These features are calculated as the ratio of the sum of duration of the notes in each category to the sum of the duration of all notes. Next, the 6 statistics are calculated for notes in each of the existing categories, i.e., for short notes duration: SNDRmean (mean value of SNDR), etc.

Ratios of Note Duration Transitions. Ratios of Note Duration Transitions (RNDT). Transitions to Longer Notes Ratio (TLNR), Transitions to Shorter Notes Ratio (TSNR) and Transitions to Equal Length Notes Ratio (TELNR). Besides measuring the duration of notes, a second extractor captures how these durations change at each note transition. Here, we check if the current note increased or decreased in length when compared to the previous. For example, regarding the TLNR metric, a note is considered longer than the previous if there is a difference of more than 10 percent 560 in length (with a minimum of $20 \mathrm{msec}$ ), as in (6). Similar cal- 561 culations apply to the TSNR and TELNR features. 562

$$
T L N R=\frac{\sum_{i=1}^{N-1}\left[n d_{i+1} / n d_{i}-1>0.1\right]}{N-1}
$$

\subsubsection{Musical Texture Features}

To the best of our knowledge, musical texture is the musical 567 concept with less directly related audio features available 568 (Section 3). However, some studies have demonstrated that 569 it can influence emotion in music either directly or by inter- 570 acting with other concepts such as tempo and mode [42]. We 571 propose features related with the music layers of a song. 572 Here, we use the sequence of multiple frequency estimates to 573 measure the number of simultaneous layers in each frame of 574 the entire audio signal, as described in Section 3.4.1. 575

Musical Layers (ML) statistics. As abovementioned, a num- 576 ber of multiple F0s are estimated from each frame of the song 577 clip. Here, we define the number of layers in a frame as the 578 number of obtained multiple F0s in that frame. Then, we 579 compute the 6 usual statistics regarding the distribution of 580 musical layers across frames, i.e., MLmean, MLstd, etc. 581

Musical Layers Distribution (MLD). Here, the number of $f 0582$ estimates in a given frame is divided into four classes: i) no 583 layers; ii) a single layer; iii) two simultaneous layers; iv) and 584 three or more layers. The percentage of frames in each of 585 these four classes is computed, measuring, as an example, 586 the percentage of song identified as having a single layer 587 (MLD1). Similarly, we compute $M L D 0, M L D 2$ and $M L D 3 . \quad 588$

Ratio of Musical Layers Transitions (RMLT). These features 589 capture information about the changes from a specific musi- 590 cal layer sequence to another (e.g., ML1 to ML2). To this 591 end, we use the number of different fundamental frequen- 592 cies (f0s) in each frame, identifying consecutive frames with 593 distinct values as transitions and normalizing the total value 594 by the length of the audio segment (in secs). Moreover, we 595 also compute the length in seconds of the longest segment 596 for each musical layer.

\subsubsection{Expressivity Features}

Few of the standard audio features studied are primarily 599 related with expressive techniques in music. However, com- 600 mon characteristics such as vibrato, tremolo and articulation 601 methods are commonly used in music, with some works 602 linking them to emotions [43]-[45].

Articulation Features. Articulation is a technique affecting 604 the transition or continuity between notes or sounds. To 605 compute articulation features, we start by detecting legato 606 (i.e., connected notes played "smoothly") and staccato (i.e., 607 short and detached notes), as described in Algorithm 1. 608 Using this, we classify all the transitions between notes in 609 the song clip and, from them, extract several metrics such 610 as: ratio of staccato, legato and other transitions, longest 611 sequence of each articulation type, etc.

In Algorithm 1, the employed threshold values were set 613 experimentally. Then, we define the following features: 614

Staccato Ratio (SR), Legato Ratio (LR) and Other Transitions 615 Ratio (OTR). These features indicate the ratio of each 616 
articulation type (e.g., staccato) to the total number of transitions between notes.

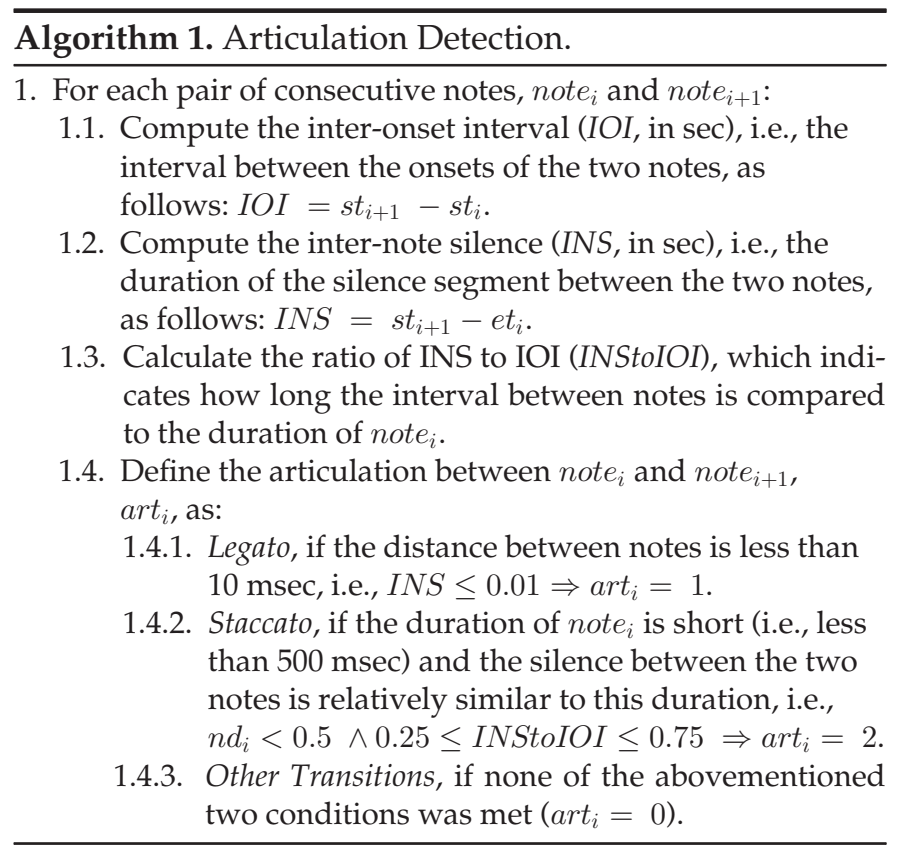

Staccato Notes Duration Ratio (SNDR), Legato Notes Duration Ratio (LNDR) and Other Transition Notes Duration Ratio (OTNDR) statistics. Based on the notes duration for each articulation type, several statistics are extracted. The first is the ratio of the duration of notes with a specific articulation to the sum of the duration of all notes. Eq. 7 illustrates this procedure for staccato $(S N D R)$. Next, the usual 6 statistics are calculated.

$$
S N D R=\frac{\sum_{i=1}^{N-1}\left[a r t_{i}=1\right] \cdot n d_{i}}{\sum_{i=1}^{N-1} n d_{i}}
$$

Glissando Features. Glissando is another kind of expressive articulation, which consists in the glide from one note to another. It is used as an ornamentation, to add interest to a piece and thus may be related to specific emotions in music.

We extract several glissando features such as glissando presence, extent, length, direction or slope. In cases where two distinct consecutive notes are connected with a glissando, the segmentation method applied (mentioned in Section 3.4.1) keeps this transition part at the beginning of the second note [40]. The climb or descent, of at least 100 cents, might contain spikes and slight oscillations in frequency estimates, followed by a stable sequence. Given this, we apply the following algorithm:

Then, we define the following features.

Glissando Presence (GP). A song clip contains glissando if any of its notes has glissando, as in (8).

$$
G P=\left\{\begin{array}{ll}
1, & \text { if } \exists i \in\{1,2, \ldots, N\}: g p_{i}=1 \\
0, & \text { otherwise }
\end{array} .\right.
$$

Glissando Extent (GE) statistics. Based on the glissando extent of each note, $g e_{i}$ (see Algorithm 2), we compute the usual 6 statistics for notes containing glissando.
Glissando Duration (GD) and Glissando Slope (GS) statistics. 674 As with GE, we also compute the same 6 statistics for glis- 675 sando duration, based on $g d_{i}$ and slope, based on $g s_{i}$ (see 676 Algorithm 2).

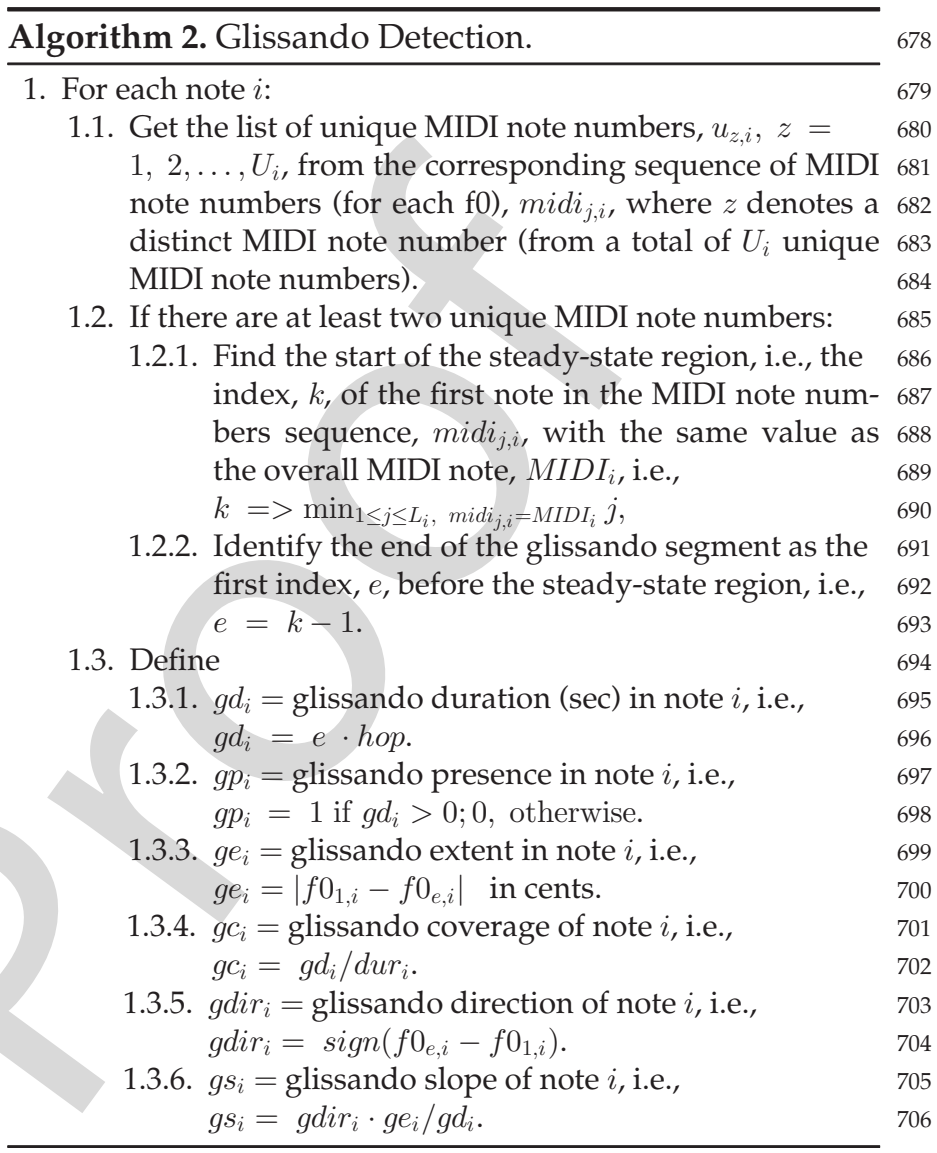

Glissando Coverage (GC). For glissando coverage, we com- 707 pute the global coverage, based on $g c_{i}$, using (9).

$$
G C=\frac{\sum_{i=1}^{N} g c_{i} \cdot n d_{i}}{\sum_{i=1}^{N} n d_{i}}
$$

Glissando Direction (GDIR). This feature indicates the 712 global direction of the glissandos in a song, (10): 713

$$
G D I R=\frac{\sum_{i=1}^{N} g p_{i}}{N}, \text { when } \text { gdir }_{i}=1 .
$$

Glissando to Non-Glissando Ratio (GNGR). This feature is 717 defined as the ratio of the notes containing glissando to the 718 total number of notes, as in (11):

$$
G N G R=\frac{\sum_{i=1}^{N} g p_{i}}{N} .
$$

Vibrato and Tremolo Features. Vibrato is an expressive 723 technique used in vocal and instrumental music that con- 724 sists in a regular oscillation of pitch. Its main characteristics 725 are the amount of pitch variation (extent) and the velocity 726 (rate) of this pitch variation. It varies according to different 727 music styles and emotional expression [44]. $\quad 728$

Hence, we extract several vibrato features, such as 729 vibrato presence, rate, coverage and extent. To this end, we 730 
apply a vibrato detection algorithm adapted from [46], as follows:

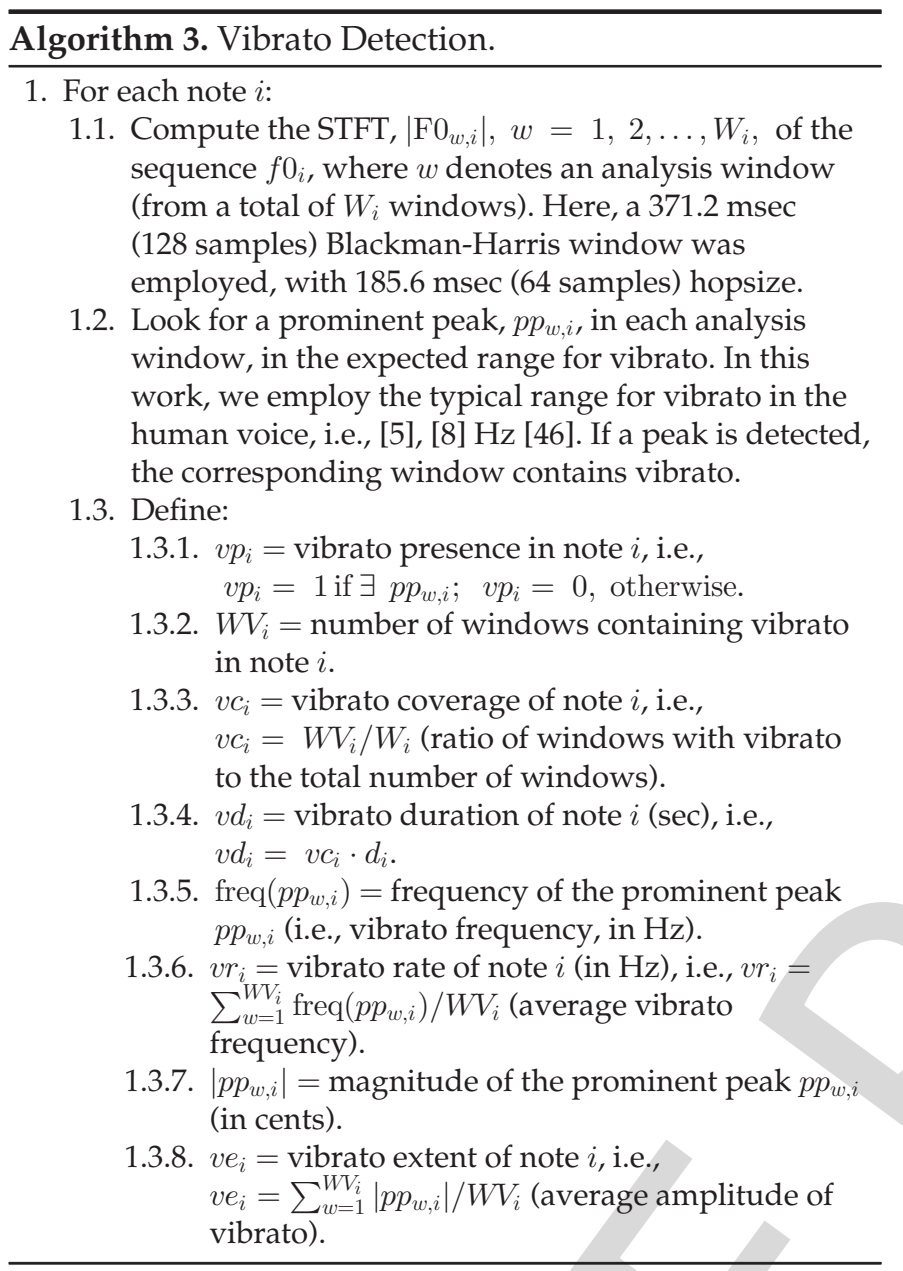

Then, we define the following features.

Vibrato Presence (VP). A song clip contains vibrato if any of its notes have vibrato, similarly to (8).

Vibrato Rate (VR) statistics. Based on the vibrato rate of each note, $v r_{i}$ (see Algorithm 3), we compute 6 statistics: VRmean, i.e., the weighted mean of the vibrato rate of each note, etc.

$$
\text { VRmean }=\frac{\sum_{i=1}^{N} v r_{i} \cdot v c_{i} \cdot n d_{i}}{\sum_{i=1}^{N} v c_{i} \cdot n d_{i}} .
$$

Vibrato Extent (VE) and Vibrato Duration (VD) statistics. As with VR, we also compute the same 6 statistics for vibrato extent, based on $v e_{i}$ and vibrato duration, based on $v d_{i}$ (see Algorithm 3).

Vibrato Coverage (VC). Here, we compute the global coverage, based on $v c_{i}$, in a similar way to (9).

High-Frequency Vibrato Coverage (HFVC). This feature measures vibrato coverage restricted to notes over note $\mathrm{C} 4$ (261.6 Hz). This is the lower limit of the soprano's vocal range [41].

Vibrato to Non-Vibrato Ratio (VNVR). This feature is defined as the ratio of the notes containing vibrato to the total number of notes, similarly to (11).

Vibrato Notes Base Frequency (VNBF) statistics. As with the $V R$ features, we compute the same 6 statistics for the base frequency (in cents) of all notes containing vibrato.
As for tremolo, this is a trembling effect, somewhat simi- 790 lar to vibrato but regarding change of amplitude. A similar 791 approach is used to calculate tremolo features. Here, the 792 sequence of pitch saliences of each note is used instead of 793 the f0 sequence, since tremolo represents a variation in 794 intensity or amplitude of the note. Given the lack of scien- 795 tific supported data regarding tremolo, we used the same 796 range employed in vibrato (i.e., $5-8 \mathrm{~Hz}$ ).

\subsubsection{Voice Analysis Toolbox (VAT) Features}

Another approach, previously used in other contexts was 799 also tested: a voice analysis toolkit.

Some researchers have studied emotion in speaking and 801 singing voice [47] and even studied the related acoustic fea- 802 tures [48]. In fact, "using singing voices alone may be effec- 803 tive for separating the "calm" from the "sad" emotion, but 804 this effectiveness is lost when the voices are mixed with 805 accompanying music" and "source separation can effec- 806 tively improve the performance" [9].

Hence, besides extracting features from the original 808 audio signal, we also extracted the same features from the 809 signal containing only the separated voice. To this end, we 810 applied the singing voice separation approach proposed by 811 Fan et al. [49] (although separating the singing voice from 812 accompaniment in an audio signal is still an open problem). 813

Moreover, we used the Voice Analysis Toolkit", a "set of 814 Matlab code for carrying out glottal source and voice qual- 815 ity analysis" to extract features directly from the audio sig- 816 nal. The selected features are related with voiced and 817 unvoiced sections and the detection of creaky voice - "a 818 phonation type involving a low frequency and often highly 819 irregular vocal fold vibration, [which] has the potential [...] 820 to indicate emotion" [50].

\subsection{Emotion Recognition}

Given the high number of features, ReliefF feature selection 823 algorithms [36] were used to select the better suited ones for 824 each classification problem. The output of the ReliefF algo- 825 rithm is a weight between -1 and 1 for each attribute, with 826 more positive weights indicating more predictive attributes. 827 For robustness, two algorithms were used, averaging the 828 weights: ReliefFequalK, where K nearest instances have 829 equal weight, and ReliefFexpRank, where K nearest instan- 830 ces have weight exponentially decreasing with increasing 831 rank. From this ranking, we use the top N features for classi- 832 fication testing. The best performing $\mathrm{N}$ indicates how many 833 features are needed to obtain the best results. To combine 834 baseline and novel features, a preliminary step is run to 835 eliminate novel features that have high correlation with 836 existing baseline features. After this, the resulting feature 837 set (baseline+novel) is used with the same ranking proce- 838 dure, obtaining a top $\mathrm{N}$ set (baseline+novel) that achieves 839 the best classification result.

As for classification, in our experiments we used Support 841 Vector Machines (SVM) [51] to classify music based on the 4842 emotion quadrants. Based on our work and in previous 843 MER studies, this technique proved robust and performed 844 generally better than other methods. Regarding kernel 845 selection, a common choice is a Gaussian kernel (RBF), 846

5. https://github.com/jckane/Voice_Analysis_Toolkit 
TABLE 3

Results of the Classification by Quadrants

\begin{tabular}{lccc}
\hline Classifier & Feat. set & \# Features & F1-Score \\
\hline SVM & baseline & 70 & $\mathbf{6 7 . 5 \%} \pm \mathbf{0 . 0 5}$ \\
SVM & baseline & 100 & $67.4 \% \pm 0.05$ \\
SVM & baseline & 800 & $71.7 \% \pm 0.05$ \\
SVM & baseline+novel & 70 & $74.7 \% \pm 0.05$ \\
SVM & baseline+novel & 100 & $\mathbf{7 6 . 4 \%} \pm \mathbf{0 . 0 4}$ \\
SVM & baseline+novel & 800 & $74.8 \% \pm 0.04$ \\
\hline
\end{tabular}

while a polynomial kernel performs better in a small subset of specific cases. In our preliminary tests RBF performed better and hence was the selected kernel.

All experiments were validated with repeated stratified 10-fold cross validation [52] (using 20 repetitions) and the average obtained performance is reported.

\section{Results ANd Discussion}

Several classification experiments were carried out to measure the importance of standard and novel features in MER problems. First, the standard features, ranked with ReliefF, were used to obtain a baseline result. Followingly, the novel features were combined with the baseline and also tested, to assess whether the results are different and statistically significant.

\subsection{Classification Results}

A summary of the attained classification results is presented in Table 3. The baseline features attained 67.5 percent F1Score (macro weighted) with SVM and 70 standard features. The same solution achieved a maximum of 71.7 percent with a very high number of features (800). Adding the novel features (i.e., standard + novel features) increased the maximum result of the classifier to 76.4 percent $(0.04$ standard deviation), while using a considerably lower number of features (100 instead of 800). This difference is statistically significant (at $\mathrm{p}<0.01$, paired T-test).

The best result (76.4 percent) was obtained with 29 novel and 71 baseline features, which demonstrates the relevance of adding novel features to MER, as will be discussed in the next section. In the paragraphs below, we conduct a more comprehensive feature analysis.

Besides showing the overall classification results, we also analyse the results obtained in each individual quadrant (Table 4), which allows us to understand which emotions are more difficult to classify and what is the influence of the standard and novel features in this process. In all our tests, a significantly higher number of songs from Q1 and Q2 were correctly classified when compared to Q3 and Q4. This seems to indicate that emotions with higher arousal are

TABLE 4

Results Per Quadrant Using 100 Features

\begin{tabular}{lccccccc}
\hline & \multicolumn{3}{c}{ baseline } & & 3 & novel \\
\cline { 2 - 4 } \cline { 6 - 8 } Quads & Prec. & Recall & F1-Score & & Prec. & Recall & F1-Score \\
\hline Q1 & $62.6 \%$ & $73.4 \%$ & $67.6 \%$ & & $74.6 \%$ & $81.7 \%$ & $78.0 \%$ \\
Q2 & $82.3 \%$ & $79.6 \%$ & $80.9 \%$ & & $88.6 \%$ & $84.7 \%$ & $86.6 \%$ \\
Q3 & $61.3 \%$ & $57.5 \%$ & $59.3 \%$ & & $71.9 \%$ & $69.9 \%$ & $70.9 \%$ \\
Q4 & $62.8 \%$ & $57.9 \%$ & $60.2 \%$ & & $69.6 \%$ & $68.1 \%$ & $68.8 \%$ \\
\hline
\end{tabular}

TABLE 5

Confusion Matrix Using the Best Performing Model.

\begin{tabular}{|c|c|c|c|c|c|}
\hline & & & predic & & \\
\hline & & Q1 & Q2 & Q3 & $Q 4$ \\
\hline & Q1 & 185.85 & 14.40 & 8.60 & 18.15 \\
\hline & Q2 & 23.95 & 190.55 & 7.00 & 3.50 \\
\hline actual & $\widetilde{Q} 3$ & 14.20 & 8.40 & 157.25 & 45.15 \\
\hline & $Q 4$ & 24.35 & 1.65 & 45.85 & 153.15 \\
\hline & Total & 246.35 & 215.00 & 218.70 & 219.95 \\
\hline
\end{tabular}

easier to differentiate with the selected features. Out of the 884 two, Q2 obtained the highest F1-Score. This goes in the 885 same direction as the results obtained in [53], and might be 886 explained by the fact that several excerpts from Q2 belong 887 to the heavy-metal genre, which has very distinctive, noise- 888 like, acoustic features.

The lower results in Q3 and Q4 (on average 12 percent 890 below the results from Q1 and Q3) can be a consequence of 891 several factors. First, more songs in these quadrants seem 892 more ambiguous, containing unclear or contrasting emo- 893 tions. During the manual validation process, we observed 894 low agreement (45.3 percent) between the subject's opinions 895 and the original AllMusic annotations. Moreover, subjects 896 reported having more difficulty distinguishing valence for 897 songs with low arousal. In addition, some songs from these 898 quadrants appear to share musical characteristics, which 899 are related to contrasting emotional elements (e.g., a happy 900 accompaniment or melody and a sad voice or lyric). This 901 concurs with the conclusions presented in [54]. 902

For the same number of features (100), the experiment 903 using novel features shows an improvement of 9 percent in 904 F1-Score when compared to the one using only the baseline 905 features. This increment is noticeable in all four quadrants, 906 ranging from 5.7 percent in quadrant 2, where the baseline 907 classifier performance was already high, to a maximum 908 increment of 11.6 percent in quadrant 3, which was the least 909 performing using only baseline features. Overall, the novel 910 features improved the classification generally, with a 911 greater influence in songs from Q3.

912

Regarding the misclassified songs, analyzing the confu- 913 sion matrix (see Table 5, averaged for the 20 repetitions of 10- 914 fold cross validation) shows that the classifier is slightly 915 biased towards positive valence, predicting more frequently 916 songs from quadrants 1 and 4 (466.3, especially Q1 with 917 246.35) than from 2 and 3 (433.7). Moreover, a significant 918 number of songs were wrongly classified between quadrants 919 3 and 4, which may be related with the ambiguity described 920 previously [54]. Based on this, further MER research needs 921 to tackle valence in low arousal songs, either by using new 922 features to capture musical concepts currently ignored or by 923 combining other sources of information such as lyrics.

\subsection{Feature Analysis}

Fig. 2 presents the total number of standard and novel audio 926 features extracted, organized by musical concept. As dis- 927 cussed, most are tonal features, for the reasons pointed out 928 previously.

As abovementioned, the best result (76.4 percent, Table 3) 930 was obtained with 29 novel and 71 baseline features, which 931 demonstrates the relevance of the novel features to MER. 


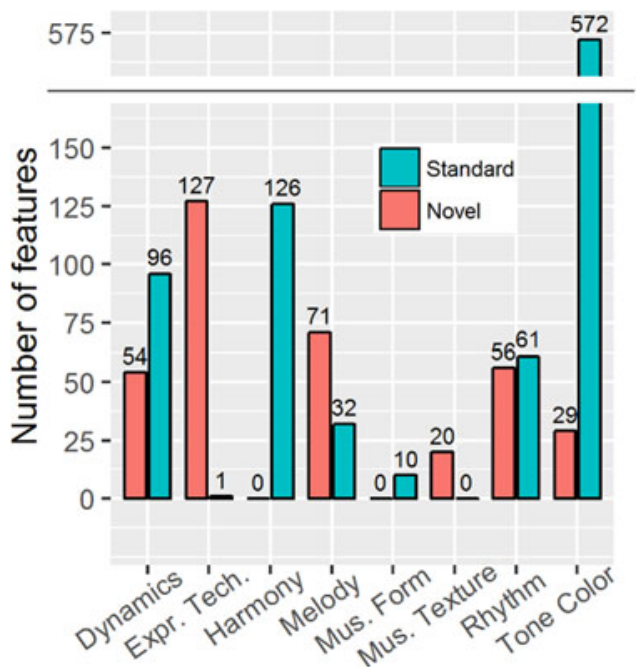

Fig. 2. Feature distribution across musical concepts.
Moreover, the importance of each audio feature was measured using ReliefF. Some of the novel features proposed in this work appear consistently in the top 10 features for each problem and many others are in the first 100, demonstrating their relevance to MER. There are also features that, while alone may have a lower weight, are important to specific problems when combined with others.

In this section we discuss the best features to discriminate each specific quadrant from the others, according to specific feature rankings (e.g., ranking of features to separate Q1 songs from non-Q1 songs). The top 5 features to discriminate each quadrant are presented in Table 6.

Except for quadrant 1, the top5 features for each quadrant contain a majority of tone color features, which are overrepresented in comparison to the remaining. It is also relevant to highlight the higher weight given by ReliefF to the top5 features of both Q2 and Q4. This difference in weights explains why less features are needed to obtain 95 percent of the maximum score for both quadrants, when compared to Q1 and Q3.

Musical texture information, namely the number of musical layers and the transitions between different texture types (two of which were extracted from voice only signals) were also very relevant for quadrant 1 , together with several rhythmic features. However, the ReliefF weight of these features to Q1 is lower when compared with the top features of other quadrants. Happy songs are usually energetic, associated with a "catchy" rhythm and high energy. The higher number of rhythmic features used, together with texture and tone color (mostly energy metrics) support this idea. Interestingly, creaky voice detection extracted directly from voice is also highlighted (it ranked $15^{\text {th }}$ ), which has previously been associated with emotion [50].

The best features to discriminate Q2 are related with tone color, such as: roughness - capturing the dissonance in the song; rolloff and MFCC - measuring the amount of high frequency and total energy in the signal; and spectral flatness measure - indicating how noise-like the sound is.

Other important features are tonal dissonance (dynamics) and expressive techniques such as vibrato. Empirically, it makes sense that characteristics like sensory dissonance, high energy, and complexity are correlated to tense, aggressive
TABLE 6

Top 5 Features for Each Quadrant Discrimination

\begin{tabular}{|c|c|c|c|c|}
\hline Q & Feature & Type & Concept & Weight \\
\hline \multirow{5}{*}{ Q1 } & $\begin{array}{l}\text { FFT Spectrum - Spectral } \\
\text { 2nd Moment (median) }\end{array}$ & base & Tone Color & 0.1467 \\
\hline & $\begin{array}{l}\text { Transitions ML1 -> } \\
\text { ML0 (Per Sec) }\end{array}$ & novel & Texture & 0.1423 \\
\hline & MFCC1 (mean) & base & Tone Color & 0.1368 \\
\hline & $\begin{array}{l}\text { Transitions ML0 -> } \\
\text { ML1 (Per Sec) }\end{array}$ & novel (voice) & Texture & 0.1344 \\
\hline & Fluctuation (std) & base & Rhythm & 0.1320 \\
\hline \multirow{5}{*}{ Q2 } & $\begin{array}{l}\text { FFT Spectrum - Spectral } \\
\text { 2nd Moment (median) }\end{array}$ & base & Tone Color & 0.2528 \\
\hline & Roughness (std) & base & Tone Color & 0.2219 \\
\hline & Rolloff (mean) & base & Tone Color & 0.2119 \\
\hline & MFCC1 (mean) & base & Tone Color & 0.2115 \\
\hline & $\begin{array}{l}\text { FFT Spectrum - Average } \\
\text { Power Spectrum (median) }\end{array}$ & base & Tone Color & 0.2059 \\
\hline \multirow{5}{*}{ Q3 } & Spectral Skewness (std) & base & Tone Color & 0.1775 \\
\hline & $\begin{array}{l}\text { FFT Spectrum - Skewness } \\
\text { (median) }\end{array}$ & base & Tone Color & 0.1573 \\
\hline & $\begin{array}{l}\text { Tremolo Notes in } \\
\text { Cents (Mean) }\end{array}$ & novel & Tremolo & 0.1526 \\
\hline & $\begin{array}{l}\text { Linear Spectral } \\
\text { Pairs } 5 \text { (std) }\end{array}$ & base & Tone Color & 0.1517 \\
\hline & MFCC1 (std) & base & Tone Color & 0.1513 \\
\hline \multirow{5}{*}{ Q4 } & $\begin{array}{l}\text { FFT Spectrum - Skewness } \\
\text { (median) }\end{array}$ & base & Tone Color & 0.1918 \\
\hline & Spectral Skewness (std) & base & Tone Color & 0.1893 \\
\hline & Musical Layers (Mean) & novel & Texture & 0.1697 \\
\hline & Spectral Entropy (std) & base & Tone Color & 0.1645 \\
\hline & Spectral Skewness (max) & base & Tone Color & 0.1637 \\
\hline
\end{tabular}

music. Moreover, research supports the association of vibrato 975 and negative energetic emotions such as anger [47]. 976

In addition to the tone color features related with the 977 spectrum, the best 20 features for quadrant 3 also include 978 the number of musical layers (texture), spectral dissonance, 979 inharmonicity (harmony), and expressive techniques such 980 as tremolo. Moreover, nine features used to obtain the maxi- 981 mum score are extracted directly from the voice-only signal. 982 Of these, four are related with intensity and loudness varia- 983 tions (crescendos, decrescendos); two with melody (vocal 984 ranges used); and three with expressive techniques such as 985 vibratos and tremolo. Empirically, the characteristics of the 986 singing voice seem to be a key aspect influencing emotion 987 in songs from quadrants 3 and 4, where negative emotions 988 (e.g., sad, depressed) usually have not so smooth voices, 989 with variations in loudness (dynamics), tremolos, vibratos 990 and other techniques that confer a degree of sadness [47] 991 and unpleasantness.

The majority of the employed features were related with 993 tone color, where features capturing vibrato, texture and 994 dynamics and harmony were also relevant, namely spectral 995 metrics, the number of musical layers and its variations, 996 measures of the spectral flatness (noise-like). More features 997 are needed to better discriminate Q3 from Q4, which musi- 998 cally share some common characteristics such as lower 999 tempo, less musical layers and energy, use of glissandos 1000 and other expressive techniques.

A visual representation of the best 30 features to distin- 1002 guish each quadrant, grouped by categories, is represented 1003 in Fig. 3. As previously discussed, a higher number of tone 1004 


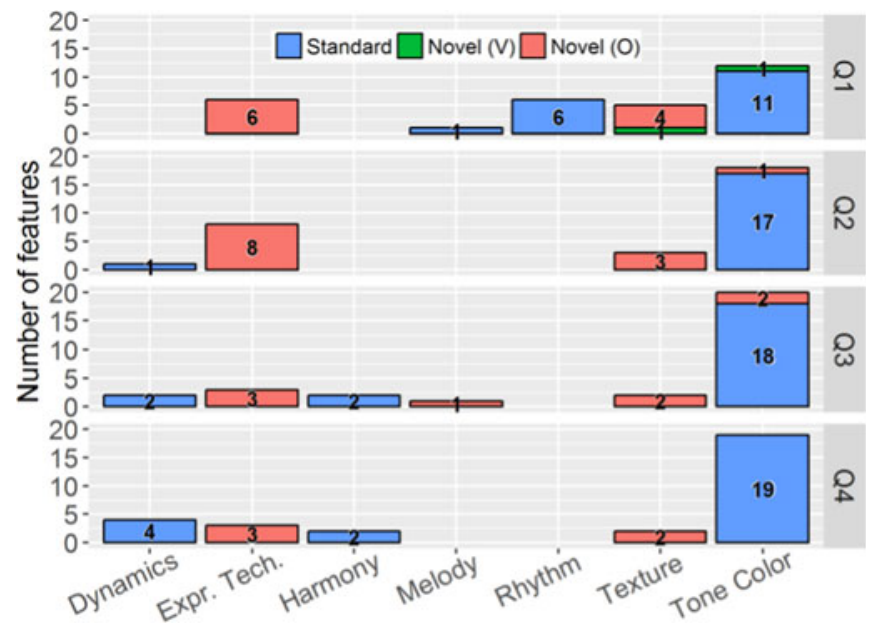

Fig. 3. Best 30 features to discriminate each quadrant, organized by musical concept. Novel $(\mathrm{O})$ are extracted from the original audio signal, while Novel (V) are extracted from the voice-separated signal.

color features is used to distinguish each quadrant (against the remaining). On the other hand, some categories of features are more relevant to specific quadrants, such as rhythm and glissando (part of the expressive techniques) for Q1, or voice characteristics to Q3.

\section{CONCLUSIONS AND FUtURE WORK}

This paper studied the influence of musical audio features in MER applications. The standard audio features available in known frameworks were studied and organized into eight musical categories. Based on this, we proposed novel more towards higher level musical concepts audio features to help bridge the identified gaps in the state-of-the-art and break the current glass ceiling. Namely, features related with musical expressive performance techniques (e.g., vibrato, tremolo, and glissando) and musical texture, which were the two less represented musical concepts in existing MER implementations. Some additional audio features that may further improve the results, e.g., features related with musical form, are still to be developed.

To evaluate our work, a new dataset was built semi-automatically, containing 900 song entries and respective metadata (e.g., title, artist, genre and mood tags), annotated according to the Russell's emotion model quadrants.

Classification results show that the addition of the novel features improves the results from 67.4 percent to 76.4 percent when using a similar number of features (100), or from 71.7 percent if 800 baseline features are used.

Additional experiments were carried out to uncover the importance of specific features and musical concepts to discriminate specific emotional quadrants. We observed that, in addition to the baseline features, novel features, such as the number of musical layers (musical texture) and expressive techniques metrics, such as tremolo notes or vibrato rates, were relevant. As mentioned, the best result was obtained with 29 novel features and 71 baseline features, which demonstrates the relevance of this work.

In the future, we will further explore the relation between the voice signal and lyrics by experimenting with multimodal MER approaches. Moreover, we plan to study emotion variation detection and to build sets of interpretable rules providing a more readable characterization of how musical features influence emotion, something that lacks when black- 1046 box classification methods such as SVMs are employed.

\section{ACKNOWLEDGMENTS}

This work was supported by the MOODetector project 1049 (PTDC/EIA-EIA/102185/2008), financed by the Fundação 1050 para Ciência e a Tecnologia (FCT) and Programa Operacio- 1051 nal Temático Factores de Competitividade (COMPETE) - 1052 Portugal, as well as the PhD Scholarship SFRH/BD/91523/ 1053 2012, funded by the Fundação para Ciência e a Tecnologia 1054 (FCT), Programa Operacional Potencial Humano (POPH) 1055 and Fundo Social Europeu (FSE). The authors would also 1056 like to thank the reviewers for their comments that helped 1057 improving the manuscript.

\section{REFERENCES}

[1] Y. Feng, Y. Zhuang, and Y. Pan, "Popular music retrieval by 1060 detecting mood," in Proc. 26th Annu. Int. ACM SIGIR Conf. Res. 1061 Dev. Inf. Retrieval, vol. 2, no. 2, pp. 375-376, 2003.

[2] C. Laurier and P. Herrera, "Audio music mood classification 1063 using support vector machine," in Proc. 8th Int. Society Music Inf. 1064 Retrieval Conf., 2007, pp. 2-4.

[3] L. Lu, D. Liu, and H.-J. Zhang, "Automatic mood detection and 1066 tracking of music audio signals," IEEE Trans. Audio Speech Lang. 1067 Process., vol. 14, no. 1, pp. 5-18, Jan. 2006.

[4] A. Flexer, D. Schnitzer, M. Gasser, and G. Widmer, "Playlist gen- 1069 eration using start and end songs," in Proc. 9th Int. Society Music 1070 Inf. Retrieval Conf., 2008, pp. 173-178.

[5] R. Malheiro, R. Panda, P. Gomes, and R. P. Paiva, "Emotionally- 1072 relevant features for classification and regression of music lyrics," 1073 IEEE Trans. Affect. Comput., 2016, doi: 10.1109/TAFFC.2016.2598569. 1074

[6] R. Panda, R. Malheiro, B. Rocha, A. Oliveira, and R. P. Paiva, 1075 "Multi-modal music emotion recognition: A new dataset, method- 1076 ology and comparative analysis," in Proc. 10th Int. Symp. Comput. 1077 Music Multidisciplinary Res., 2013, pp. 570-582.

[7] O. Celma, P. Herrera, and X. Serra, "Bridging the music semantic 1079 gap," in Proc. Workshop Mastering Gap: From Inf. Extraction Seman- 1080 tic Representation, 2006, vol. 187, no. 2, pp. 177-190.

[8] Y. E. Kim, E. M. Schmidt, R. Migneco, B. G. Morton, P. Richard- 1082 son, J. Scott, J. A. Speck, and D. Turnbull, "Music emotion recogni- 1083 tion: A state of the art review," in Proc. 11th Int. Society Music Inf. 1084 Retrieval Conf., 2010, pp. 255-266.

[9] X. Yang, Y. Dong, and J. Li, "Review of data features-based music 1086 emotion recognition methods," Multimed. Syst., pp. 1-25, Aug. 2017, 1087 https://link.springer.com/article/10.1007/s00530-017-0559-4 1088

[10] Y.-H. Yang, Y.-C. Lin, Y.-F. Su, and H. H. Chen, "A regression 1089 approach to music emotion recognition," IEEE Trans. Audio. 1090 Speech. Lang. Processing, vol. 16, no. 2, pp. 448-457, Feb. 2008.

[11] C. Laurier, "Automatic classification of musical mood by content- 1092 based analysis," Universitat Pompeu Fabra, 2011, http://mtg.upf. 1093 edu/node/2385

[12] T. Bertin-Mahieux, D. P. W. Ellis, B. Whitman, and P. Lamere, 1095 "The million song dataset," in Proc. 12th Int. Society Music Inf. 1096 Retrieval Conf., 2011, pp. 591-596.

[13] J. A. Russell, "A circumplex model of affect," J. Pers. Soc. Psychol., vol. 39, no. 6, pp. 1161-1178, 1980.

[14] K. Hevner, "Experimental studies of the elements of expression in music," Am. J. Psychol., vol. 48, no. 2, pp. 246-268, 1936.

[15] H. Katayose, M. Imai, and S. Inokuchi, "Sentiment extraction in 1102 music," in Proc. 9th Int. Conf. Pattern Recog., 1988, pp. 1083-1087. 1103

[16] R. Panda and R. P. Paiva, "Using support vector machines for 1104 automatic mood tracking in audio music," in Proc. 130th Audio 1105 Eng. Society Conv., vol. 1, 2011, Art. no. 8378.

[17] M. Malik, S. Adavanne, K. Drossos, T. Virtanen, D. Ticha, and R. 1107 Jarina, "Stacked convolutional and recurrent neural networks for 1108 music emotion recognition," in Proc. 14th Sound $\mathcal{E}$ Music Comput. 1109 Conf., 2017, pp. 208-213.

[18] N. Thammasan, K. Fukui, and M. Numao, "Multimodal fusion of 1111 EEG and musical features music-emotion recognition," in Proc. 1112 31st AAAI Conf. Artif. Intell., 2017, pp. 4991-4992.

[19] A. Aljanaki, Y.-H. Yang, and M. Soleymani, "Developing a bench- 1114 mark for emotional analysis of music," PLoS One, vol. 12, no. 3, 1115 Mar. 2017, Art. no. e0173392. 
[20] A. Gabrielsson and E. Lindström, "The influence of musical structure on emotional expression," in Music and Emotion, vol. 8, New York, NY, USA: Oxford University Press, 2001, pp. 223-248.

[21] C. Laurier, O. Lartillot, T. Eerola, and P. Toiviainen, "Exploring relationships between audio features and emotion in music," in Proc. 7th Triennial Conf. Eur. Society Cognitive Sciences Music, vol. 3, 2009, pp. 260-264.

[22] A. Friberg, "Digital audio emotions - An overview of computer analysis and synthesis of emotional expression in music," in Proc. 11th Int. Conf. Digital Audio Effects, 2008, pp. 1-6.

[23] O. C. Meyers, A mood-based music classification and exploration system. MIT Press, 2007.

[24] O. Lartillot and P. Toiviainen, "A Matlab toolbox for musical feature extraction from audio," in Proc. 10th Int. Conf. Digital Audio Effects (DAFx), 2007, pp. 237-244, https://dspace.mit.edu/ handle/1721.1/39337

[25] G. Tzanetakis and P. Cook, "MARSYAS: A framework for audio analysis," Organised Sound, vol. 4, no. 3, pp. 169-175, 2000.

[26] D. Cabrera, S. Ferguson, and E. Schubert, "'Psysound3': Software for acoustical and psychoacoustical analysis of sound recordings," in Proc. 13th Int. Conf. Auditory Display, 2007, pp. 356-363.

[27] H. Owen, Music Theory Resource Book. London, UK: Oxford University Press, 2000.

[28] L. B. Meyer, Explaining Music: Essays and Explorations. Berkeley, CA, USA: University of California Press, 1973

[29] Y. E. Kim, E. M. Schmidt, and L. Emelle, "Moodswings: A collaborative game for music mood label collection," in Proc. 9th Int. Society Music Inf. Retrieval Conf., 2008, pp. 231-236.

[30] A. Aljanaki, F. Wiering, and R. C. Veltkamp, "Studying emotion induced by music through a crowdsourcing game," Inf. Process. Manag., vol. 52, no. 1, pp. 115-128, Jan. 2016.

[31] X. Hu, J. S. Downie, C. Laurier, M. Bay, and A. F. Ehmann, "The 2007 MIREX audio mood classification task: Lessons learned," in Proc. 9th Int. Society Music Inf. Retrieval Conf., 2008, pp. 462-467.

[32] P. Vale, "The role of artist and genre on music emotion recognition," Universidade Nova de Lisboa, 2017

[33] J. S. Downie, X. Hu, and J. S. Downie, "Exploring mood metadata: Relationships with genre, artist and usage metadata," in Proc. 8th Int. Society Music Inf. Retrieval Conf., 2007, pp. 67-72.

[34] A. B. Warriner, V. Kuperman, and M. Brysbaert, "Norms of valence, arousal, and dominance for 13,915 English lemmas," Behav. Res. Methods, vol. 45, no. 4, pp. 1191-1207, Dec. 2013.

[35] M. M. Bradley and P. J. Lang, "Affective norms for English words (ANEW): Instruction manual and affective ratings," Psychology, vol. Technical, no. C-1, p. 0, 1999.

[36] M. Robnik-Sikonja and I. Kononenko, "Theoretical and empirical analysis of ReliefF and RReliefF," Mach. Learn., vol. 53, no. 1-2, pp. 23-69, 2003.

[37] E. Benetos, S. Dixon, D. Giannoulis, H. Kirchhoff, and A. Klapuri, "Automatic music transcription: Challenges and future directions," I. Intell. Inf. Syst., vol. 41, no. 3, pp. 407-434, 2013.

[38] J. Salamon and E. Gómez, "Melody extraction from polyphonic music signals using pitch contour characteristics," IEEE Trans. Audio. Speech. Lang. Processing, vol. 20, no. 6, pp. 1759-1770, Aug. 2012.

[39] K. Dressler, "Automatic transcription of the melody from polyphonic music," Ilmenau University of Technology, 2016.

[40] R. P. Paiva, T. Mendes, and A. Cardoso, "Melody detection in polyphonic musical signals: Exploiting perceptual rules, note salience, and melodic smoothness," Comput. Music J., vol. 30, no. 4, pp. 80-98, Dec. 2006.

[41] A. Peckham, J. Crossen, T. Gebhardt, and D. Shrewsbury, The Contemporary Singer: Elements of Vocal Technique. Berklee Press, 2010.

[42] G. D. Webster and C. G. Weir, "Emotional responses to music: interactive effects of mode, texture, and tempo," Motiv. Emot., vol. 29, no. 1, pp. 19-39, Mar. 2005, https://link.springer.com/ article/10.1007\%2Fs11031-005-4414-0

[43] P. Gomez and B. Danuser, "Relationships between musical structure and psychophysiological measures of emotion," Emotion, vol. 7, no. 2, pp. 377-387, May 2007.

[44] C. Dromey, S. O. Holmes, J. A. Hopkin, and K. Tanner, "The effects of emotional expression on vibrato," J. Voice, vol. 29, no. 2, pp. 170-181, Mar. 2015.

[45] T. Eerola, A. Friberg, and R. Bresin, "Emotional expression in music: Contribution, linearity, and additivity of primary musical cues," Front. Psychol., vol. 4, 2013, Art. no. 487.

[46] J. Salamon, B. Rocha, and E. Gómez, "Musical genre classification using melody features extracted from polyphonic music signals," in IEEE Int. Conf. Acoustics Speech Signal Process., 2012, pp. 81-84.
[47] K. R. Scherer, J. Sundberg, L. Tamarit, and G. L. Salomão, 1195 "Comparing the acoustic expression of emotion in the speaking 1196 and the singing voice," Comput. Speech Lang., vol. 29, no. 1, 1197 pp. 218-235, Jan. 2015.

[48] F. Eyben, G. L. Salomão, J. Sundberg, K. R. Scherer, and B. W. 1199 Schuller, "Emotion in the singing voice-A deeperlook at acoustic 1200 features in the light ofautomatic classification," EURASIP J. Audio 1201 Speech Music Process., vol. 2015, no. 1, Dec. 2015, Art. no. 19.

1202

[49] Z.-C. Fan, J.-S. R. Jang, and C.-L. Lu, "Singing voice separation 1203 and pitch extraction from monaural polyphonic audio music via 1204 DNN and adaptive pitch tracking," in Proc. IEEE 2nd Int. Conf. 1205 Multimedia Big Data, 2016, pp. 178-185.

[50] A. Cullen, J. Kane, T. Drugman, and N. Harte, "Creaky voice and 1207 the classification of affect," in Proc. Workshop Affective Social Speech 1208 Signals, 2013, http://tcts.fpms.ac.be/ drugman/Publi_long/ 1209

[51] C.-C. Chang and C.-J. Lin, "LIBSVM: A library for support vector 1210 machines," ACM Trans. Intell. Syst. Technol., vol. 2, no. 3, pp. 1-27, 1211 Apr. 2011.

[52] R. O. Duda, Peter E. Hart, and D. G. Stork, Pattern Classification. Hoboken, NJ, USA: Wiley, 2000.

. "Heavy metal music and emotional dysphoria among listeners," Psychol. Pop. Media Cult 1216 vol. 2, no. 2, pp. 74-85, 2013.

[54] Y. Hong, C.-J. Chau, and A. Horner, "An analysis of low-arousal 1218 piano music ratings to uncover what makes calm and sad music 1219 so difficult to distinguish in music emotion recognition," J. Audio 1220 Eng. Soc., vol. 65, no. 4, 2017.

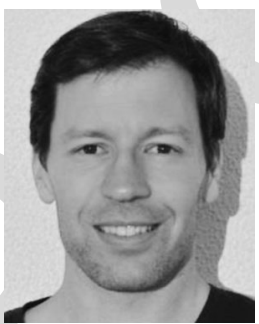

Renato Panda received the bachelor's and mas- 1222 ter's degrees in automatic mood tracking in audio 1223 music from the University of Coimbra. He is work- 1224 ing toward the PhD degree in the Department of 1225 Informatics Engineering, University of Coimbra. 1226 $\mathrm{He}$ is a member of the Cognitive and Media 1227 Systems research group at the Center for Infor- 1228 matics and Systems of the University of Coimbra 1229 (CISUC). His main research interests include 1230 music emotion recognition, music data mining 1231 and music information retrieval (MIR). In October 1232 2012, he was the main author of an algorithm that performed best in the 1233 MIREX 2012 Audio Train/Test: Mood Classification task, at ISMIR'2012. 1234

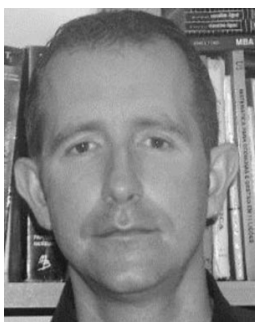

Ricardo Malheiro received the bachelor's and mas- 1235 ter's degrees (Licenciatura - five years) in informat- 1236 ics engineering and mathematics (branch of 1237 computer graphics) from the University of Coimbra. 1238 $\mathrm{He}$ is working toward the $\mathrm{PhD}$ degree at the Univer- 1239 sity of Coimbra. He is a member of the Cognitive 1240 and Media Systems research group at the Center 1241 for Informatics and Systems of the University of 1242 Coimbra (CISUC). His main research interests 1243 include natural language processing, detection of 1244 emotions in music lyrics and text and text/data min- 1245 ing. He teaches at Miguel Torga Higher Institute, Department of Informatics. 1246 Currently, he is teaching decision support systems, artificial intelligence and 1247 data warehouses and big data.

1248

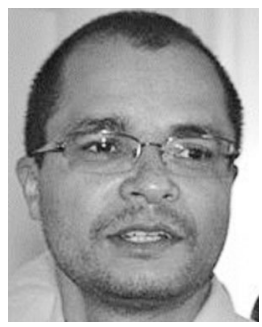

Rui Pedro Paiva received the bachelor's, mas- 1249 ter's (Licenciatura - 5 years) and doctoral degrees 1250 in informatics engineering from the University of 1251 Coimbra, in 1996, 1999, 2007, respectively. He is 1252 a professor with the Department of Informatics 1253 Engineering, University of Coimbra. He is a mem- 1254 ber of the Cognitive and Media Systems research 1255 group at the Center for Informatics and Systems 1256 of the University of Coimbra (CISUC). His main 1257 research interests include music data mining, 1258 music information retrieval (MIR) and audio proc- 1259 essing for clinical informatics. In 2004, his algorithm for melody detection 1260 in polyphonic audio won the ISMIR'2004 Audio Description Contest - mel- 1261 ody extraction track, the 1st worldwide contest devoted to MIR methods. 1262 In October 2012, his team developed an algorithm that performed best in 1263 the MIREX 2012 Audio Train/Test: Mood Classification task.

$\triangleright$ For more information on this or any other computing topic, 1266 please visit our Digital Library at www.computer.org/publications/dlib. 1267 